\title{
Proton Linacs for Boron Neutron Capture Therapy
}

\author{
Arlene J. Lennox \\ Fermi National Accelerator Laboratory \\ P.O. Box 500, Batavia, Illinois 60510
}

August 1993

Presented at the 1993 Particle Accelerator Conference (CBEAF), Washington, D.C., May 17-20, 1993 


\section{Disclaimer}

This report was prepared as an account of work sponsored by an agency of the United States Government. Neither the United States Government nor any agency thereof, nor any of their employees, makes any warranty, express or implied, or assumes any legal liability or responsibility for the accuracy, completeness, or usefulness of any information, apparatus, product, or process disclosed, or represents that its use would not infringe privately owned rights. Reference herein to any specific commercial product, process, or service by trade name, trademark, manufacturer, or otherwise, does not necessarily constitute or imply its endorsement, recommendation, or favoring by the United States Government or any agency thereof. The views and opinions of authors expressed herein do not necessarily state or reflect those of the United States Government or any agency thereof. 


\title{
Proton Linacs for Boron Neutron Capture Therapy
}

\author{
Arlene J. Lennox \\ Fermi National Accelerator Laboratory* and Rush University \\ P.O. Box 500, MS 301, Batavia, Illinois 60510-0500 USA
}

\begin{abstract}
Recent advances in the ability to deliver boron-containing drugs to brain tumors have generated interest in $-4 \mathrm{MeV}$ linacs as sources of epithermal neutrons for radiation therapy. In addition, fast neutron therapy facilities have been studying methods to moderate their beams to take advantage of the high cross section for epithermal neutrons on boron-10. This paper describes the technical issues involved in each approach and presents the motivation for undertaking such studies using the Fermilab linac. The problems which must be solved before therapy can begin are outlined. Status of preparatory work and results of preliminary measurements are presented.
\end{abstract}

\section{INTRODUCTION}

Despite the efforts of many researchers, the prognosis for a patient diagnosed with an inoperable advanced brain tumor (glioma) is dismal. The difficulty with using radiation therapy is that the tumor propagates by sending out microscopic clusters of cells close to the main body of the tumor. Even when the gross tumor is killed by radiation these clusters continue to grow. If a large margin is included around the gross tumor it is possible to destroy the clusters, but the process causes unacceptable damage to the healthy tissue in which the clusters are embedded. Hence, it is advantageous to sensitize the tumor cells to radiation without sensitizing the healthy cells. One way to sensitize the tumor is to introduce into the body a compound containing boron-10. Depending on the exact nature of the carrier, boron-10 will be absorbed by various parts of the body, including the tumor. However, healthy brain tissue will not absorb the compound because it is protected by the blood-brain barrier. When the brain is exposed to neutron radiation the large cross section for interactions between thermal neutrons and boron- 10 will result in a larger dose to tumor than healthy tissue. This form of radiation therapy is called boron neutron capture therapy (BNCT).

In recent years the Department of Energy has been supporting research in the development of boron pharmaceuticals, and a number of drugs are ready or nearly ready for clinical trials. In addition, several reactors have been modified to provide neutrons for early clinical trials [1] and work is proceeding on a tandem cascade accelerator [2]. However, much remains to be done in developing accelerator sources needed to make BNCT more widely available. Because of the high currents needed to achieve adequate dose rates, proton linacs are well suited for this application.

*Operated by the Universities Research Association, Inc., under contract No. DE-AC02-76CHO3000 with the U. S. Department of Energy.

\section{PRODUCTION NEAR THRESHOLD}

The present consensus is that the optimum neutron energy for BNCT is in tens of keV. In this energy range neutrons will penetrate the skull and be thermalized by the time they reach the tumor. One approach is to produce neutrons at an energy as close as possible to the $\mathrm{keV}$ range in order to minimize the need for moderating material. Recent progress in the technology of radiofrequency quadrupole (RFQ) linacs and the $1.8 \mathrm{MeV}$ production threshold for the ${ }^{7} \mathrm{Li}(\mathrm{p}, \mathrm{n}){ }^{7} \mathrm{Be}$ reaction have led to the hope that a $2.5 \mathrm{MeV}$ RFQ could accelerate protons which would strike a lithium target to produce the neutrons[3]. Despite a great deal of interest in this approach, the critical problems remain unsolved. Estimates of the average proton current required for acceptable dose rates range from 5 to 30 milliamperes [2],[4]. This uncertainty is largely due to uncertainties in the relative biological effectiveness (RBE) of neutrons in tissue at these energies. Current requirements decrease with increasing RBE, but until at least one prototype is constructed the RBE's cannot be measured. In addition, a moderating system must be incorporated to accommodate the fact that the neutrons produced in this reaction are too energetic. A number of moderating schemes have been proposed but they cannot be evaluated until an accelerator becomes available. Finally, the problem of cooling the lithium target is unlikely to be solved within reasonable economic constraints, and the safety issues related to using lithium in a clinical environment are formidable.

The difficulties associated with a lithium target have led to interest in using a beryllium target. Beryllium has a higher melting point than lithium, is easier to cool, and has been used successfully in clinical fast neutron therapy facilities. The neutron production threshold for protons impinging on a beryllium target is $2.2 \mathrm{MeV}$ and the yield becomes comparable to a lithium target yield at about $4 \mathrm{MeV}$. Using $4 \mathrm{MeV}$ protons and a beryllium target produces even more energetic neutrons than the system described above. Monte Carlo studies indicate that the spectrum can be moderated to reduce the high energy component [5] but no work is being done on designing a $4 \mathrm{MeV}$ proton source for BNCT. It is believed that a $4 \mathrm{MeV}$ RFQ is impractical and that a drift tube linac (DTL) is required. Use of a DTL with a beryllium target will solve the technical issues associated with the target but the questions relating to specifying an appropriate proton current remain unanswered. The most economical approach is to identify an existing $4 \mathrm{MeV}$ accelerator and use it and a prototype moderating system to measure RBE's and dose rates. Such experiments would provide the data needed to extrapolate to the DTL operating parameters with confidence. This step is critical for the continued evolution of compact neutron sources for BNCT. 


\section{SPALLATION SOURCES}

The targeting schemes described above attempt to produce neutrons at low energies to minimize moderation requirements at the expense of requiring high primary beam currents. An alternate approach is to build a spallation source for which relatively low currents produce large neutron fluxes at high energies requiring a great deal of moderation. Researchers at Paul Scherrer Institute have been using a 72 $\mathrm{MeV}$ cyclotron to test target and moderating systems [6]. They have the advantage of access to an existing accelerator and have actually used a number of heavy targets with various moderating schemes to measure neutron spectra and photon contamination. Their 80 microampere beam is believed adequate to produce an acceptable dose rate but a $70 \mathrm{MeV}$ linac operating at about 200 microamperes would be an ideal proton source for this approach. Operating parameters for a multipurpose $70 \mathrm{MeV}$ medical proton linac have been described elsewhere [7].

\section{FAST NEUTRON THERAPY BOOST}

The neutron therapy facility (NTF) at Fermilab uses a Be target and $66 \mathrm{MeV}$ protons from a DTL to produce fast neutrons for radiation therapy. NTF conducted clinical trials treating gliomas with fast neutrons from 1979 until 1989. In many cases patients experienced remarkable, but temporary improvement. Autopsies showed that neutrons were successful in killing the tumors and that the patients died from necrosis of nearby healthy tissue [8]. With the existing fast neutron beam is was not possible to establish a therapeutic window for a long-term cure - that is, enough dose to destroy the tumor without causing unacceptable damage to neighboring healthy tissue. However, it is possible that a dose enhancement of as little as 10 or $20 \%$ from the introduction of boron into the tumor would open the therapeutic window.

The cross sections for neutron ${ }^{10} \mathrm{~B}$ interactions decrease with increasing energy, so it is not expected that boron would enhance the dose due to the higher energy neutrons. However, preliminary phantom studies at NTF using Fricke dosimetry and gold foils show a peak in the thermal component at 5 to $10 \mathrm{~cm}$ in tissue. These studies also show that introduction of one inch of iron into the beam has little effect on the thermal component at shallow depths but reduces it by $20 \%$ deeper in tissue [9]. Further studies must be done to measure the absolute thermal flux and to determine whether an iron filter can be used to limit the thermal dose to distal healthy tissue. One possibility for enhancing the thermal component is to introduce $20-30 \mathrm{~cm}$ of $\mathrm{D}_{2} 0$ into the beam. In evaluating this technique one must consider not only the resulting energy spectrum but the possible loss of collimating ability. Fast neutron therapy relies on collimation to reduce healthy tissue dose. Epithermal and thermal beams scatter throughout the entire brain regardless of upstream collimation. Hence, the goal is to shift the NTF energy spectrum downward to enhance the probability of thermalization in tissue while perturbing the beam's angular distribution as little as possible.

Previous attempts to measure the energy spectrum of this beam have been hampered by the $200 \mathrm{MHz}$ beam structure. The beam arrives at detectors in one-nanosecond micropulses, five nanoseconds apart for a total of 57 microseconds, and this pattern is repeated 15 times per second. This structure and the $\sim 8$ meter neutron flight path have precluded the use of time-of-flight techniques. In addition, the types of detectors normally used with Bonner spheres experience saturation effects with these instantaneous dose rates. To address this issue studies are being conducted on a beam chopper which would allow individual micropulses to be accelerated through the linac at a $15 \mathrm{~Hz}$ rate [10]. These studies include not only the design of the chopper but also the installation of diagnostics which are sensitive to the relatively low currents transmitted by the chopper. Once the chopper is working the energy spectrum will be measured and it will be possible to use existing computer codes to design an energy degrader.

In addition to measuring the energy spectrum it is necessary to develop dosimetry techniques to study the relative contribution of fast and thermal neutrons to the total dose as a function of depth in a phantom. This must be done for the existing beam so that it can be compared to a newly designed degraded beam. Techniques will include foil activation studies, Fricke dosimetry, measurements with miniature tissue equivalent detectors and appropriate thermoluminescent detector systems. Finally, biophysical studies with boronated cell culture systems and rodent tumors must be done to check the efficacy of using boron- 10 to enhance tumor dose from the NTF beam. Similar biophysical studies at a fast neutron facility in Essen have shown the dose enhancement in animals to be greater than expected from calculations based on conventional dosimetry [11]. Hence, the results of the biophysical studies are very important in establishing a prescribed dose to humans.

\section{CONCLUSIONS}

Present schemes for accelerator-produced neutron beams for BNCT use trade-offs between lower yield reactions with neutron energies as close as possible to epithermal energies and higher yield reactions with higher energy neutrons. Proton linacs play an important role in both scenarios. However, before linacs can be used for BNCT a great deal of work must be done. In some cases it would be good to use data from existing lower current accelerators to obtain the information needed to design practical systems.

\section{REFERENCES}

[1] Rolf F. Barth, Albert H. Soloway, Ralph G. Fairchild and Robert M. Brugger, "Boron Neutron Capture Therapy for Cancer", Cancer, Vol. 70, No. 12, 1992.

[2] R. E. Shefer, R. E. Klinkowstein, J.C. Yanch and G.L.Brownell, "An Epithermal Neutron Source for BNCT Using a Tandem Cascade Accelerator," in Progress in Neutron Capture Therapy for Cancer, B. J. Allen, D.E. Moore and B. V. Harrington, eds., Plenum Press, New York, 1992, pp. 119-122.

[3] M. A. Lone, A. M. Ross, J. S. Fraser, S. O. Schriber, S. A. Kushneriuk, and W. N. Selander, "Low Energy 
${ }^{7} \mathrm{Li}(\mathrm{p}, \mathrm{n}){ }^{7} \mathrm{Be}$ Neutron Source (Canutron)," Chalk River Laboratories, Internal Report AECL-7413, April, 1982.

[4] T. P. Wangler, J. E. Stovall, T. S. Bhatia, C. K. Wang, T. E. Blue, and R. A. Gahbauer, "Conceptual Design of an RFQ Accelerator-Based Neutron Source for Boron Neutron-Capture Therapy," Proceedings of the 1989 IEEE Particle Accelerator Conference, IEEE \#89CH26690, pp. 678-680.

[5] C-K. Chris Wang and Brian R. Moore, "On the Study of Energy Spectra and Angular Distributions of the Neutrons Emitted from a Beryllium Target Bombarded with 4-MeV Protons for Neutron Capture Therapy," Proceedings of the Fifth International Symposium on Neutron Capture Therapy, Columbus, Ohio, 1992.

[6] J.F. Crawford, H. Reist, H. Conde, K. Elmgren, T. Roennqvist, E.Grusell, B. Nilsson, O. Pettersson, P. Stromberg and B. Larsson, "Neutrons for Capture Therapy Produced by $72 \mathrm{MeV}$ Protons," in Progress in Neutron Capture Therapy for Cancer, B. J. Allen, D. E.
Moore and B. V. Harrington, eds., Plenum Press, New York, 1992, pp. 129-132.

[7] Arlene J. Lennox, "Hospital-based proton linear accelerator for particle therapy and radioisotope production," Nucl. Instr. and Meth. B56/57 (1991) pp. 1197-1200.

[8] K. R. Saroja, JoAnne Mansell, F. R. Hendrickson, Lionel Cohen, and Arlene Lennox, "Failure of Accelerated Neutron Therapy to Control High Grade Astrocytomas," Int. J. Radiation Oncology Biol. Phys. Vol 17 pp. 1295-1297.

[9] L. Wielopolski, unpublished.

[10] T. Kroc, private communication.

[11] Wolfgang Sauerwein, Proceedings of the Fifth International Symposium on Neutron Capture Therapy, Columbus, Ohio, 1992. 